\author{
ANNALES \\ POLONICI MATHEMATICI \\ XLI (1983)
}

\title{
Biholomorphic invariance of capacity and the capacity of an annulus
}

\author{
by JeRzy Kalina (Łódź)
}

\begin{abstract}
In this paper we prove the biholomorphic invariance of the capacity defined in [6] and demonstrate the existence of an extremal function for condensers biholomorphically equivalent to an annulus. We are also concerned with a conjecture of S. S. Chern, H. I. Levine and L. Nirenberg [3] and we give a partial positive answer to it.
\end{abstract}

Introduction. Let $D$ be a bounded domain in $C^{n}$ whose boundary consists of two components which are $(2 n-1)$-dimensional smooth differentiable manifolds. We denote by $\operatorname{Adm} D$ the class of $C^{2}$-smooth real valued function on $\bar{D}$ which are supposed to be $C^{2}$-plurisubharmonic on $D$ and equal zero on one component of the boundary and one on the other component. In our considerations we assume that $\operatorname{Adm} D \neq \emptyset$. We call the domain $D$ a condenser.

Let us define the functional

$$
\operatorname{Adm} D \ni u \mapsto J(u)=\int_{D} d u \wedge d^{c} u \wedge\left[d d^{c} u\right]^{n-1} \in R .
$$

With any condenser $D$ we associate the real number

$$
\operatorname{Cap} D=\inf _{u \in A d m D} J(u)
$$

which is called its capacity.

In the present paper a theorem on the invariance of capacities under biholomorphic mappings is proved. We demonstrate also the existence of an extremal function $u$ for condensers biholomorphically equivalent to an annulus, whence, in particular, follows that two annuli with a different ratio of radii are not biholomorphically equivalent. Finally, we are concerned with the conjecture of Chern, Levine and Nirenberg [3] in the case where $D=D_{1}-\bar{D}_{0}, D_{0}$ and $D_{1}$ are assumed to be strictly pseudoconvex. In the case where $\operatorname{Adm} D$ contains an extremal function, a positive answer was obtained by Bedford and Taylor [1]. 
1. Biholomorphic invariance of capacity. To begin with we introduce

Definition 1. Two condensers $D$ and $D^{*}$ are called biholomorphically equivalent if there exists a diffeomorphism $f: \bar{D} \rightarrow \bar{D}^{*}$ such that $\bar{f}=f \mid D$ is a biholomorphic mapping between $D$ and $D^{*}$.

Our further considerations will be concerned with the existence property of an extremal function. By an extremal function in the class $\operatorname{Adm} D$ we mean any $u^{*} \in \operatorname{Adm} D$ such that

$$
\inf _{u \in A \operatorname{din} D} J(u)=J\left(u^{*}\right)
$$

It turns out [6] that $u^{*} \in \operatorname{Adm} D$ is an extremal function if and only if

$$
\left[d d^{c}\left(u^{*} \mid D\right)\right]^{n}=0 \text {. }
$$

Lemma 1. If two condensers $D$ and $D^{*}$ are biholomorphically equivalent, then the existence of an extremal function in $\operatorname{Adm} D$ implies the existence in $\operatorname{Adm} D^{*}$ and conversely.

Proof. The proof is trivial and follows from standard calculations.

Let $P(R ; r)=\left\{z \in C^{n}: r<|z|^{2}<R, 0<r<R\right\}$. Then we have

Lemma 2. $P(R ; r)$ is a condenser and the class $\operatorname{Adm} P(R ; r)$ contains an extremal function.

Proof. Trivially, $P(R ; r)$ is a condenser. By simple calculations we may also verify that

$$
u(z)=[\ln (R / r)]^{-1} \ln \left(|z|^{2} / r\right), \quad z \in \bar{P}(R ; r),
$$

is an extremal function.

We are going to prove

THEOREM 1. Two condensers which are biholomorphically equivalent have equal capacities.

Proof. Let $D$ and $D^{*}$ be two condensers which are biholomorphically equivalent, and let $f$ be a mapping which establishes the equivalence between $D$ and $D^{*}$. Denote by $w=\left(w^{1}, \ldots, w^{n}\right)$ and $z=\left(z^{1}, \ldots, z^{n}\right)$ coordinate systems in $D^{*}$ and $D$, respectively. If $u \in \operatorname{Adm} D$, then $u^{*}$ $=u \circ f^{-1} \in \operatorname{Adm} D^{*}$. By simple calculations we see that

(5) $d\left(u^{*} \mid D^{*}\right) \wedge d^{c}\left(u^{*} \mid D^{*}\right)$

$$
=2 i \sum_{i, p, k, s=1}^{n}\left(u_{\mid i} \circ(f \mid D)^{-1} \bar{u}_{\mid \bar{k}} \circ(f \mid D)^{-1}\right) z_{\mid s}^{l} z_{\mid \bar{p}}^{k} d w^{s} \wedge d \bar{w}^{p},
$$

$$
d d^{c}\left(u^{*} \mid D^{*}\right)=2 i \sum_{i, p, k, s=1}^{n}\left(u_{\mid p \bar{k}} \circ(f \mid D)^{-1}\right) z_{\mid s}^{p} \bar{z}_{\mid i}^{k} d w^{s} \wedge d \bar{w}^{i} .
$$

Taking into account (5) and (6), we obtain 


$$
\begin{aligned}
J^{*}\left(u^{*}\right) & =\int_{D^{*}} d u^{*} \wedge d^{c} u^{*} \wedge\left[d d^{c} u^{*}\right]^{n-1} \\
& =c \int_{D^{*}}\left(\sum_{i, k=1}^{n} u_{\mid i} u_{\mid \bar{k}} M_{i k}\right) \circ(f \mid D)^{-1}|d z / d w|^{2} d V^{*},
\end{aligned}
$$

where $c=2^{2 n}(n-1) !, d z / d w$ is the Jacobian matrix of $(f \mid D)^{-1}, M_{i k}$ $=(-1)^{i}(-1)^{k} m_{i k}$, and $m_{i k}$ is $(i, k)$-th minor matrix of $\left[u_{|j| \bar{k}}\right]_{1 \leqslant i, k \leqslant n}$. It is easy to see that (7) is equivalent to

$$
J^{*}\left(u^{*}\right)=J(u) .
$$

By the definition of capacity there are sequences $\left\{u_{n}\right\} \subset \operatorname{Adm} D$ and $\left\{u_{n}^{*}\right\} \subset \operatorname{Adm} D$ such that $J\left(u_{n}\right) \rightarrow \operatorname{Cap} D$ and $J^{*}\left(u_{n}^{*}\right) \rightarrow \operatorname{Cap} D^{*}$ for $n \rightarrow x$. Consider the functions $\tilde{u}_{n}^{*}=u_{n} \circ f^{-1} \in \operatorname{Adm} D^{*}$ and $\tilde{u}_{n}=u_{n}^{*} \circ f \in \operatorname{Adm} D$. By (8) we have $J\left(\tilde{u}_{n}\right)=J^{*}\left(u_{n}^{*}\right)$ and $J^{*}\left(u_{n}^{*}\right)=J\left(u_{n}\right)$ for $n=1,2, \ldots$ Therefore

$$
\begin{aligned}
& \operatorname{Cap} D=\lim _{n \rightarrow x} J\left(u_{n}\right)=\lim _{n \rightarrow x} J^{*}\left(\tilde{u}_{n}^{*}\right) \geqslant \operatorname{Cap} D^{*}, \\
& \operatorname{Cap} D \leqslant \lim _{n \rightarrow x} J\left(\tilde{u}_{n}\right)=\lim _{n \rightarrow x} J^{*}\left(u_{n}^{*}\right)=\operatorname{Cap} D^{*},
\end{aligned}
$$

which shows that $\operatorname{Cap} D=\operatorname{Cap} D^{*}$ and proves the lemma.

Let $R^{*}>r^{*}>0$ and $R>r>0$ be such that $R^{*} / r^{*} \neq R / r$. We have

THEOREM 2. The condensers $P\left(R^{*} ; r^{*}\right)$ and $P(R ; r)$ are not biholomorphically equivalent.

Proof. By Lemma 2 it follows that

$$
u(z)=[\ln (R / r)]^{-1} \ln \left(|z|^{2} / r\right) \quad \text { for } z \in \bar{P}(R ; r)
$$

and

$$
u^{*}(z)=\left[\ln \left(R^{*} / r^{*}\right)\right]^{-1} \ln \left(|z|^{2} / r^{*}\right) \quad \text { for } z \in \bar{P}\left(R^{*} ; r^{*}\right)
$$

are extremal in $\operatorname{Adm} P(R ; r)$ and $\operatorname{Adm} P\left(R^{*} ; r^{*}\right)$, respectively. In the case of an annulus we may easily compute the capacities involved:

$$
\begin{aligned}
\operatorname{Cap} P(R ; r) & =\int_{P(R: r)} d u \wedge d^{c} u \wedge\left[d d^{c} u\right]^{n-1} \\
& =\int_{P(R: r)} d\left(u d^{c} u \wedge\left[d d^{c} u\right]^{n-1}\right)-\int_{P(R ; r)} u\left[d d^{c} u\right]^{n} \\
& =\int_{r P(R: r)} u d^{c} u \wedge\left[d d^{c} u\right]^{n-1}=\int_{C_{1}} d^{c} u \wedge\left[d d^{c} u\right]^{n-1} \\
& =[\ln (R / r)]^{-n} \int_{C_{1}} d^{c} \ln |z|^{2} \wedge\left[d d^{c} \ln |z|^{2}\right]^{n-1} \\
& \equiv[\ln (R / r)]^{-n} \Gamma
\end{aligned}
$$

and likewise 


$$
\text { Cap } P\left(R^{*} ; r^{*}\right)=\left[\ln \left(R^{*} / r^{*}\right)\right]^{-n} \Gamma^{*} \text {. }
$$

We may assume that $R^{*}>R$; by the Stokes theorem we have

$$
\Gamma^{*}-\Gamma=\int_{c_{\mathrm{i}}^{*}-c_{\mathrm{t}}} d^{c} \ln |z|^{2} \wedge\left[d d^{c} \ln |z|^{2}\right]^{n-1}=\int_{P\left(R^{*} ; R\right)}\left[d d^{c} \ln |z|^{2}\right]^{n}=0
$$

From (9), (10) and (11) we obtain

$$
\text { Cap } P\left(R^{*} / r^{*}\right) / \operatorname{Cap} P(R ; r)=[\ln (R / r)]^{n} /\left[\ln \left(R^{*} / r^{*}\right)\right]^{n} \neq 1 \text {. }
$$

In view of Theorem 1 this proves the desired result.

We end the section with two remarks:

Remark 1. For every condenser $D=D_{1} \backslash \bar{D}_{0}$ the domain $D_{1}$ is a domain of holomorphy. If there exists some $u \in \operatorname{Adm} D$ such that $u(x) \neq 0$ for $x \in D$, then $D_{0}$ is also a domain of holomorphy.

Remark 2. Let $D=D_{1} \backslash \bar{D}_{0}$ and $\tilde{D}=\tilde{D}_{1} \backslash \tilde{D}_{0}$ be two condensers which are biholomorphically equivalent. Then $D_{1}$ and $\tilde{D}_{1}$ are also biholomorphically equivalent.

EXAMPLE. We shall give an example of a condenser which is not biholomorphically equivalent to an annulus.

Let $D_{1}$ be a strictly pseudoconvex domain which is supposed to be not biholomorphically equivalent to a ball. We also assume that $D_{1}$ has the compact closure in $C^{n}$. By assumption, one can find (see [4]) a neighbourhood $U \supset \partial D_{1}$ and a strictly $C^{2}$-plurisubharmonic function $g$ such that

$$
U \cap D_{1}=\{x \in U: g(x)<0\}, \quad \partial D_{1}=\{x \in U: g(x)=0\}
$$

and $d g(x) \neq 0$ for every $x \in U$. For sufficiently small $\varepsilon>0$ the set

$$
\partial D_{\mathrm{o}} \stackrel{\mathrm{df}}{=}\{x \in U: g(x)=-\varepsilon\}
$$

is a connected $(2 n-1)$-dimensional $C^{2}$-smooth manifold and

$$
D=\{x \in U: 0>g(x)>-\varepsilon\}
$$

is a condenser (as an admissible function we may take $U(x)=1 / \varepsilon(g(x)+\varepsilon)$, $x \in \bar{D})$.

By Lemma 3 the condenser $D$ is not biholomorphically equivalent to an annulus.

2. The Chern-Levine-Nirenberg conjecture. Now, we will be concerned with the conjecture posed by Chern, Levine and Nirenberg [3].

Let $D=D_{1} \backslash \bar{D}_{0}$ be a condenser. By $\Gamma(D)$ we shall denote a class of $(2 n-1)$-dimensional closed currents (in the sense of de Rhan) in $\bar{D}$, which contains $\partial D_{1}$ and $-\partial D_{0}$ (here we assume

$$
\partial D_{k}(\varphi) \stackrel{\mathrm{dr}}{=} \int_{\partial D_{k}} i_{k}^{*} \varphi \quad \text { for } k=0,1
$$


and for every $(2 n-1)$-form $\varphi$ on $\bar{D}$, where $i_{k}$ denotes the $C^{2}$-embedding of $\partial D_{k}$ in $\bar{D}$ and $\partial D_{1}$ and $\partial D_{0}$ have orientations induced by $D$ ) such that for every $\gamma \in \Gamma(D)$ there are positive $n$-currents $G$ and $\tilde{G}$ on $\bar{D}$ (i.e. taking nonnegative values on every positive $n$-form on $\vec{D}$ ) with the properties:

$$
b G=\partial D_{1}-\gamma, \quad b \tilde{G}=\gamma+\partial D_{0},
$$

$b$ denotes the boundary operator.

Let $\Gamma^{*}(D)=\{\Gamma(D)\} \cup\{-\Gamma(D)\}$. Define the functionals

$$
\operatorname{Adm} D \ni u \mapsto R_{i}(u)=\int_{d D_{i}} d^{c} u \wedge\left[d d^{c} u\right]^{n-1} \in R \quad \text { for } i=0,1
$$

We have

Theorem 3. If a condenser $D=D_{1} \backslash \bar{D}_{0}$ is such that $D_{1}$ and $D_{0}$ are strictly pseudoconvex and

$$
\inf _{u \in A d m D} \int_{D}\left[d d^{c} u\right]^{n}=0
$$

then

$1^{0}$ the functional $R_{1}$ is convex and $R_{0}$ is concave, respectively,

$2^{\circ}$ the following equality holds:

$$
\inf _{u \in A d m D} R_{1}(u)=\left|\sup _{u \in A d m D} \inf _{\gamma \in \Gamma^{*}(D)} \int_{\gamma} d^{c} u \wedge\left[d d^{c} u\right]^{n-1}\right|=\operatorname{Cap} D .
$$

Proof. By the pseudoconvexity of $D_{1}$ and $D_{0}$ there exists a real-valued $C^{2}$-function $f$ defined in $C^{n}$ which fulfils the following conditions:

(a) $D=\left\{x \in C^{n}: f(x)<0\right\}, \partial D=\left\{x \in C^{n}: f(x)=0\right\}$,

(b) there are neighbourhoods $V_{1} \supset \partial D_{1}$ and $V_{0} \supset \partial D_{0}$ such that $f \mid V_{1}$ and $-f \mid V_{0}$ are plurisubharmonic and $d f(x) \neq 0$ for $x \in V_{0} \cup V_{1}$.

By simple calculations we have [5]

$$
\delta^{2} R_{k}(u)(h, t)=\frac{1}{2} n(n-1) \int_{\partial D_{k}} d^{c} h \wedge d d^{c} h \wedge\left[d d^{c} u(t)\right]^{n-2} \quad \text { for } k=0,1,
$$

where $h=u-\tilde{u}, u(t)=u+t h, t \in[0,1]$, and $u, \tilde{u} \in \operatorname{Adm} D$. Since $d f(x) \neq 0$ for $x \in V_{1} \cup V_{0}$, we have

$$
h(x)=f(x) r_{h}(x), \quad x \in \bar{D},
$$

where $r_{h} \in C^{1}(\bar{D})$.

Let $i_{k}: \partial D_{k} \rightarrow \bar{D}, k=0,1$ be $C^{2}$-imbeddings. Let $i_{k}^{*}: \Lambda(\bar{D}) \rightarrow \Lambda\left(\partial D_{k}\right), k$ $=0,1$ be exterior algebra homomorphisms. It is easy to check that

$$
i_{k}^{*}\left(d^{c} h \wedge d d^{c} h\right)=\left(r_{h} \circ i_{k}\right)^{2} i_{k}^{*} d^{c} f \wedge i_{k}^{*} d d^{c} f .
$$


Hence we get

$\delta^{2} R_{k}(u)(h, t)=\frac{1}{2} n(n-1) \int_{r D_{k}} r_{h}^{2} d^{c} f \wedge d d^{c} f \wedge\left[d d^{c} u(t)\right]^{n-2} \stackrel{d r}{=} \frac{1}{2} n(n-1) \int_{c D_{k}} g d S$, where $d S=* d f /\|d f\|$ is the surface area on $\partial D_{k}$, since

$$
r_{h}^{2} d f \wedge d^{c} f \wedge d d^{c} f \wedge\left[d d^{c} u(t)\right]^{n-2}=g d f \wedge(* d f /\|d f\|)
$$

and

$$
r_{h}^{2} d f \wedge d^{c} f \wedge d d^{c} f \wedge\left[d d^{c} u(t)\right]^{n-2} \geqslant 0
$$

in $\left(D_{1} \backslash \bar{D}_{0}\right) \cap V_{1},[6]$. Analogously, we have

$$
r_{h}^{2} d f \wedge d^{c} f \wedge d d^{c} f \wedge\left[d d^{c} u(t)\right]^{n-2} \leqslant 0
$$

in $\left(D_{1} \backslash \bar{D}_{0}\right) \cap V_{0}$. By the above we infer that

$$
g(x) \geqslant 0 \text { for } x \in \hat{\partial} D_{1} \text { and } g(x) \leqslant 0 \text { for } x \in \partial D_{0} .
$$

Since for every $u \in \operatorname{Adm} D$

$$
\left[d d^{c} u\right]^{n} \geqslant 0
$$

for every $\gamma \in \Gamma(D)$ and every $u \in \operatorname{Adm} D$ we have

$$
\int_{i D_{1}} d^{c} u \wedge\left[d d^{c} u\right]^{n-2} \geqslant \int_{\gamma} d^{c} u \wedge\left[d d^{c} u\right]^{n-2} \geqslant \int_{-r_{0} D_{0}} d^{c} u \wedge\left[d d^{c} u\right]^{n-2}
$$

(here $\int_{\gamma}$ is identified with $\gamma$ ).

By the same considerations as above and by the fact that $u \mid c D_{0}=0$, we obtain

$$
\int_{-i D_{0}} d^{c} u \wedge\left[d d^{c} u\right]^{n-1} \geqslant 0
$$

From (12), (13) and from the definition of $\Gamma^{*}(D)$ we infer that for every $\gamma \in \Gamma^{*}(D)$

$$
\int_{\nu} d^{c} u \wedge\left[d d^{c} u\right]^{n-1} \geqslant-\int_{i D_{1}} d^{c} u \wedge\left[d d^{c} u\right]^{n-1}
$$

From (14) it follows that

$$
\left|\sup _{u \in A d m D} \inf _{\gamma \in \Gamma^{*}(D)} \int_{\gamma} d^{c} u \wedge\left[d d^{c} u\right]^{n-1}\right|=\inf _{u \in A d m D} \int_{\lambda D_{1}} d^{c} u \wedge\left[d d^{c} u\right]^{n-1}
$$

It remains to prove the relation

$$
\inf _{u \in A \mid m D} \int_{i D_{1}} d^{c} u \wedge\left[d d^{c} u\right]^{n-1}=\operatorname{Cap} D
$$


By assumption there is a sequence $\left\{u_{k}\right\} \subset \operatorname{Adm} D$ such that

$$
\lim _{k \rightarrow x} \int_{D}\left[d d^{c} u_{k}\right]^{n}=0
$$

We will prove that

$$
\lim _{k \rightarrow x} J\left(u_{k}\right)=\operatorname{Cap} D
$$

We see that

$$
\lim _{k \rightarrow x} \delta J\left(u_{k}\right)\left(h_{k}, 0\right)=-(n+1) \lim _{k \rightarrow x} \int_{D} h_{k}\left[d d^{c} u_{k}\right]^{n}=0 .
$$

Therefore, for arbitrary $\varepsilon>0$ we may find $N(\varepsilon)$ such that for every $k>N(\varepsilon)$ we have

$$
\left|\delta J\left(u_{k}\right)\left(h_{k}, 0\right)\right|<\varepsilon, \quad h_{k}=\tilde{u}-u_{k} .
$$

Since $J$ is convex [5], it follows that

$$
J\left(u_{k}\right) \leqslant J(\tilde{u})+\varepsilon
$$

for arbitrary $\tilde{u} \in \operatorname{Adm} D$ and $k>N(\varepsilon)$. By (15) the sequence $\left\{J\left(u_{k}\right)\right\}$ is bounded and we may choose a subsequence $\left\{J\left(u_{k_{i}}\right)\right\}$ which is convergent to some $g \geqslant 0$. Hence we have

$$
g=\lim _{i \rightarrow x} J\left(u_{k_{i}}\right) \leqslant J(\tilde{u})+\varepsilon .
$$

From the arbitrariness of $\varepsilon>0$ we get

$$
g \leqslant J(\tilde{u})
$$

and then from the arbitrariness of $\tilde{u}$ we infer that

$$
g \leqslant \operatorname{Cap} D .
$$

Together with the evident inequality

$$
g \geqslant \operatorname{Cap} D
$$

this yields

$$
g=\operatorname{Cap} D .
$$

Because the last equality holds for an arbitrary convergent subsequence $\left\{u_{\boldsymbol{k}_{i}}\right\}$ we conclude that

$$
g=\lim _{k \rightarrow x} J\left(u_{k}\right)=\operatorname{Cap} D,
$$

which proves our assertion.

Using the Stokes theorem it is easy to verify that 


$$
R_{1}(u)=\int_{D} d u \wedge d^{c} u \wedge\left[d d^{c} u\right]^{n-1}-\int_{D} u\left[d d^{c} u\right]^{n}
$$

and

$$
\delta R_{1}(u)(h, t)=n\left(\int_{D} u d d^{c} h \wedge\left[d d^{c} u(t)\right]^{n-1}-\int_{D} h\left[d d^{c} u(t)\right]^{n}\right) .
$$

From (16) and (17) it follows that

$$
\lim _{k \rightarrow \infty} R_{1}\left(u_{k}\right)=\operatorname{Cap} D \text {. }
$$

Since $u d d^{c} \tilde{u} \wedge\left[d d^{c} u\right]^{n-1}$ is non-negative for every $u, \tilde{u} \in \operatorname{Adm} D$, therefore from (18) and the above remark we arrive at the inequality

$$
\lim _{k \rightarrow \infty} \inf \delta R_{1}\left(u_{k}\right)\left(h_{k}, 0\right) \geqslant 0 .
$$

Hence, for $\varepsilon>0$ there exists $N(\varepsilon)$ such that for every $k>N(\varepsilon)$ we have

$$
\delta R_{1}\left(u_{k}\right)\left(h_{k}, 0\right)>-\varepsilon, \quad h_{k}=\tilde{u}-u_{k} .
$$

By the convexity of $R_{1}$ we infer that

$$
R_{1}\left(u_{k}\right) \leqslant R_{1}(\tilde{u})+\varepsilon, \quad k>N(\varepsilon) .
$$

Using then (19) and the same arguments as in the proof of (16), we obtain

$$
\operatorname{Cap} D=\inf _{u \in A d m D} R_{1}(u),
$$

thus concluding the proof.

Applying Theorem $\mathrm{A}$ of [2] we are going to prove

THEOREM 4. If the class of admissible functions for a condenser $D$ contains an extremal function, then

$$
\begin{aligned}
\sup _{u \in \operatorname{Adm} D} \inf _{\gamma \in \Gamma^{*}(D)}\left|\int_{\gamma} d^{c} u \wedge\left[d d^{c} u\right]^{n-1}\right| & =\inf _{u \in \operatorname{Adm} D} \sup _{\gamma \in \Gamma^{*}(D)}\left|\int_{\gamma} d^{c} u \wedge\left[d d^{c} u\right]^{n-1}\right| \\
& =\operatorname{Cap} D .
\end{aligned}
$$

Proof. Since for every $\gamma \in \Gamma(D)$ and every $u \in \operatorname{Adm} D$ we have

$$
\int_{\gamma} d^{c} u \wedge\left[d d^{c} u\right]^{n-1} \geqslant 0
$$

if follows that

$$
\sup _{u \in A d m D} \inf _{\gamma \in \Gamma^{*}(D)}\left|\int_{\gamma} d^{c} u \wedge\left[d d^{c} u\right]^{n-1}\right|=\sup _{u \in A d m D} \inf _{\gamma \in \Gamma(D)} \int_{\gamma} d^{c} u \wedge\left[d d^{c} u\right]^{n-1}
$$

By the definition of $\Gamma(D)$ we have

$$
\inf _{\gamma \in \Gamma(D)} \int_{\gamma} d^{c} u \wedge\left[d d^{c} u\right]^{n-1}=\int_{-\partial D_{0}} d^{c} u \wedge\left[d d^{c} u\right]^{n-1}=-R_{0}(u)
$$


and

$$
\sup _{\gamma \in \Gamma(D)} \int_{\gamma} d^{c} u \wedge\left[d d^{c} u\right]^{n-1}=\int_{\partial D_{1}} d^{c} u \wedge\left[d d^{c} u\right]^{n-1}=R_{1}(u)
$$

Let $u^{*}$ be an extremal function in $\operatorname{Adm} D$. By Theorem $A$ [2] we have

$$
u-u^{*}=h \leqslant \dot{0} \quad \text { in } \bar{D} \text {. }
$$

Let $f$ be a $C^{2}$-smooth real-valued function such that

$$
D=\left\{x \in C^{n}: f(x)<0\right\}, \quad \partial D=\left\{x \in C^{n}: f(x)=0\right\}
$$

and

$$
d f(x) \neq 0 \quad \text { for } \quad x \in \partial D
$$

It can be easily seen that

$$
\delta\left[-R_{0}\right]\left(u^{*}\right)(h, t)=-\delta R_{0}\left(u^{*}\right)(h, t)=-n \int_{i D_{0}} d^{c} h \wedge\left[d d^{x} u(t)\right]^{n-1},
$$

where $h=u-u^{*}$. By the same arguments as in Theorem 3 we have

$$
h=u-u^{*}=r_{h} f \text {, }
$$

where $r_{h} \in C^{1}(\bar{D})$. From $(20)$ it follows that $r_{h}(x) \geqslant 0$ for $x \in \partial D_{0}$. Thus

$$
\delta\left[-R_{0}\right]\left(u^{*}\right)(h, t)=-n \int_{\partial D_{0}} r_{h} d^{c} f \wedge\left[d d^{c} u(t)\right]^{n-1} \leqslant 0
$$

for every function $u \in \operatorname{Adm} D$ and for every $t \in[0,1]$. The last inequality shows that the functional $-R_{0}$ attains its maximum for $u^{*}$. Simple calculations show also that $-R_{0}\left(u^{*}\right)=\operatorname{Cap} D$. By analogous considerations we may also prove the second part of our statement, as desired.

\section{References}

[1] E. Bedford and B. A.T a y lor, Variational properties of the complex Monge-Ampére equation, Duke Math. J. 45 (1978).

[2] - and -, The Dirichlet problem for a complex Monge-Apmére equation, Inventiones Math. 37 (1976), p. 1-44.

[3] S. S. Chern, H. 1. Levine and L. Nirenberg, Intrinsic norms on a complex manifold, Global Analysis, Papers in honor of K. Kodaira, ed. by D. C. Spencer and Iynaga, Univ. of Tokyo Press and Princeton Univ. Press, Tokyo 1969, p. 120-139.

[4] H. Grauert, Uber Modifikationen und exzeptionelle analytische Mengen, Math. Ann. 146 (1962), p. 331-368.

[5] J. Kalina, A variational characterization of condenser capacities in $C^{n}$ within a class of plurisubharmonic functions, this fascicle, p. 167-173.

[6] P. Lelong, Plurisubharmonic functions and positive differential forms, Gordon and Breach, New York 1969. 